Supporting Information

\title{
Regulatory Implications of Structural Changes in Tyr201 of Oxygen Sensor Protein FixL
}

\author{
Takeo Yamawaki ${ }^{\dagger}$, Haruto Ishikawa ${ }^{\dagger}$, Misao Mizuno ${ }^{\dagger}$, Hiro Nakamura ${ }^{\ddagger}$, \\ Yoshitsugu Shiro ${ }^{\ddagger}$, Yasuhisa Mizutani ${ }^{+*}$ \\ †Department of Chemistry, Graduate School of Science, Osaka University, 1-1 \\ Machikaneyama, Toyonaka, Osaka 560-0043, Japan. \\ ‡RIKEN SPring-8 Center, 1-1-1 Kouto, Sayo, Hyogo 679-5148, Japan. \\ *Corresponding author. E-mail: mztn@chem.sci.osaka-u.ac.jp
}



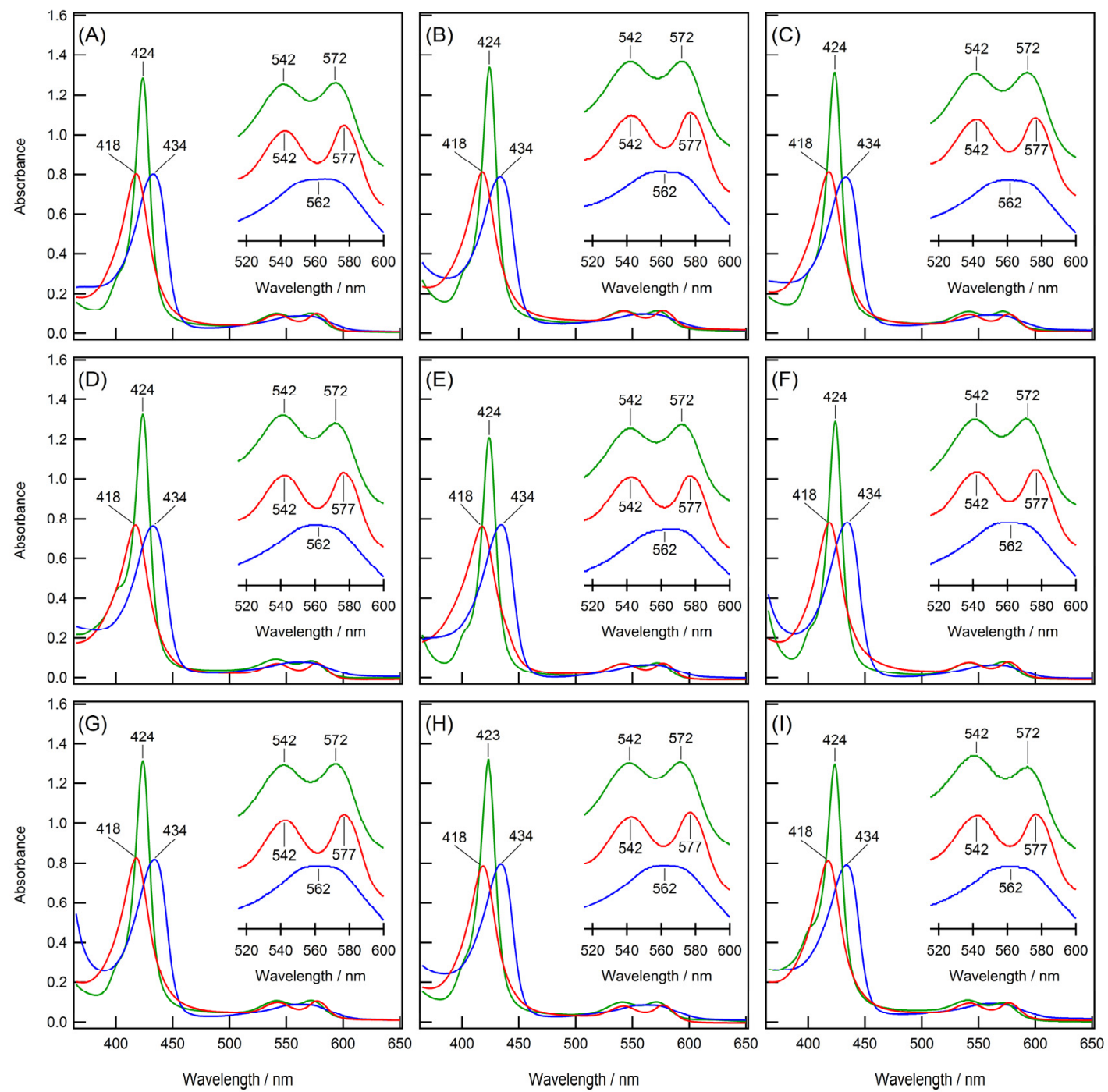

Figure S1. Absorption spectra of WT and eight mutants of FixL. Panel (A)-(I) show the spectra of WT, Y172F, Y190F, Y197F, Y201F, Y201H, Y297F, Y379F, and Y496F mutants, respectively. Blue, red, and red lines are for deoxy, oxy, and carbonmonoxy forms. 


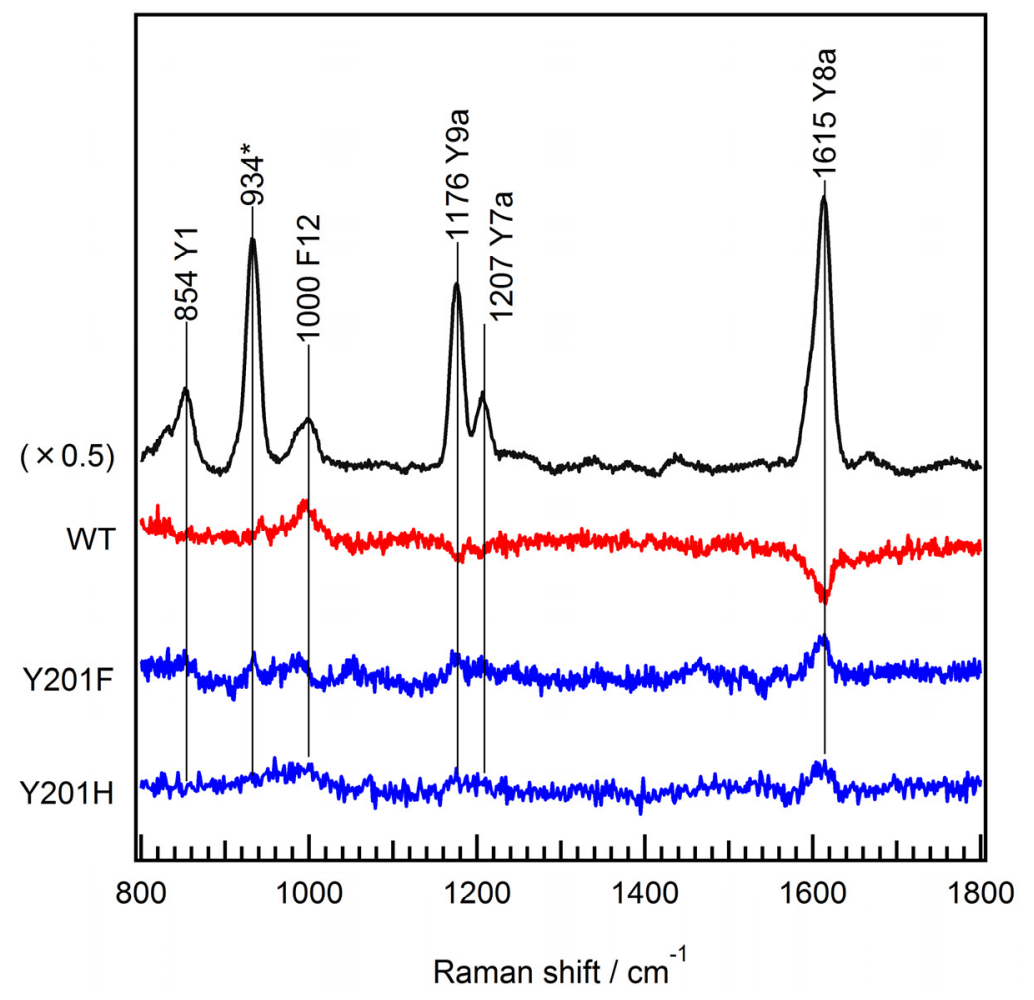

Figure S2. Difference UVRR spectra between deoxy and oxy forms of WT, Y201F, and Y201F mutants FixL. The UVRR spectrum for the oxy form of WT is shown at the top for comparison. 


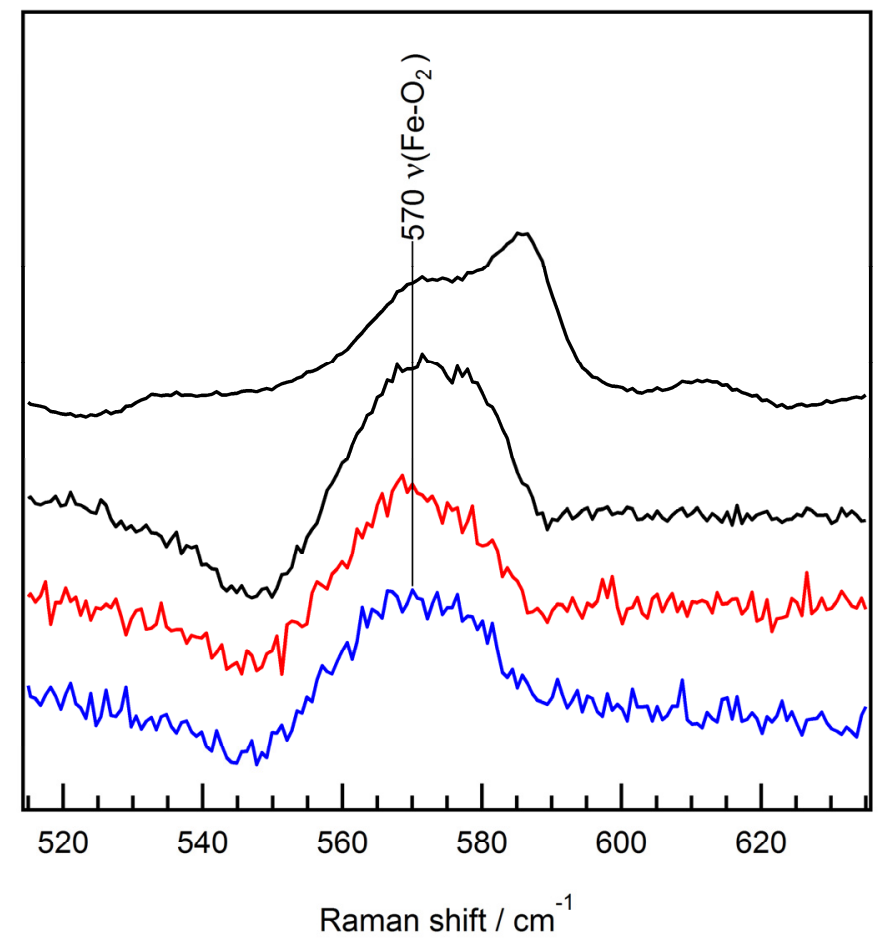

Figure S3. Difference spectra of ${ }^{16} \mathrm{O}_{2}$ - and ${ }^{18} \mathrm{O}_{2}$-derivatives for assignment of the $570-\mathrm{cm}^{-1}$ band to iron-oxygen stretching mode $\left[v\left(\mathrm{Fe}-\mathrm{O}_{2}\right)\right]$. 

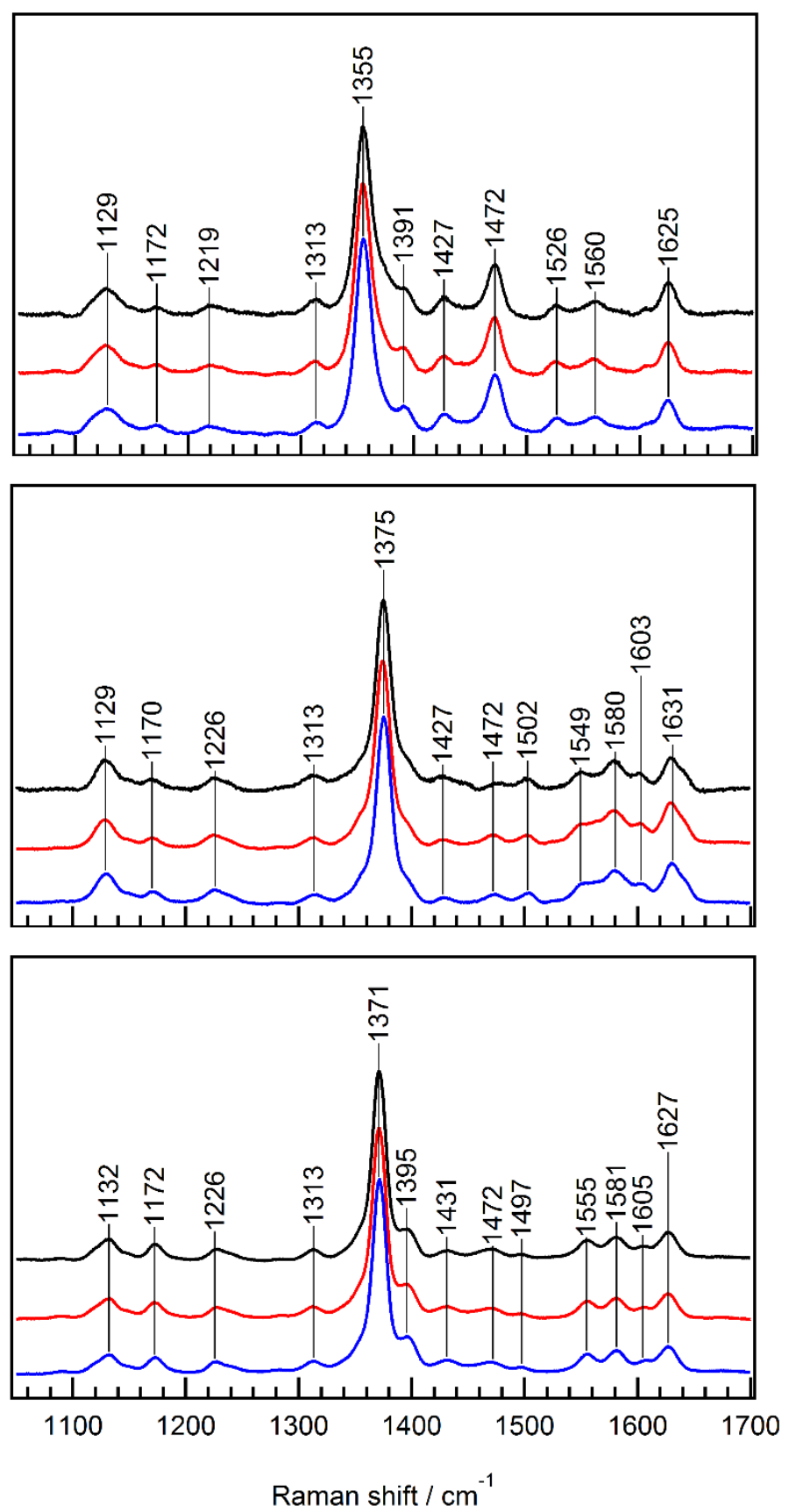

Figure S4. Visible resonance Raman spectra of deoxy (top), oxy (middle), and carbonmonoxy forms (bottom) of WT, Y201F and Y201F mutants of FixL. Black, red, and red traces show the spectra of WT, Y201F and Y201F, respectively. Raman scattering was excited by 410-nm light, which was second harmonic of output of an Nd:YLF-pumped Ti:sapphire laser (Photonics Industries, TU-L). Repetition rate and energy of the pulse were $1 \mathrm{kHz}$ and $0.5 \mu \mathrm{J}$, respectively. Accumulation time for obtaining each spectrum was $15 \mathrm{~min}$. 